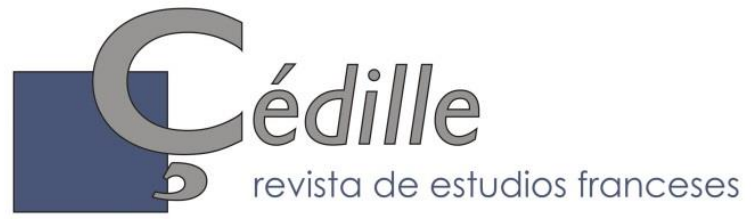

ISSN: 1699-4949

$n^{0} 18$ (otoño de 2020)

Monografías 11

Epistemocrítica: análisis literario y saber científico Amelia Gamoneda Lanza \& Francisco González Fernández, editores científicos

\title{
Performativité du droit. Efficacité de la littérature et de l'image
}

\author{
Christine Baron \\ Université de Poitiers \\ christine.baron@univ-poitiers.fr \\ ORCID : 0000-0002-3788-7683
}

\section{Resumen}

La literatura francesa parece haber abandonado el territorio de la ficción en provecho de la investigación y del testimonio. Los relatos judiciales constituyen una parte importante de ésta y el papel de estos relatos y de las imágenes fílmicas en nuestra percepción del derecho no puede ser subestimado. Hay varias maneras de concebir la relación entre derecho y literatura (o cine). La literatura y el cine dan vida a personajes de un proceso judicial, pero tienen también tendencia a imponer su interpretación de los hechos. Por último, algunos procesos se convierten en símbolos a través de la literatura y el cine; de este modo, el escritor tiene el poder de moldear nuestra concepción de la justicia.

Palabras clave: Justicia, Derecho, Literatura narrativa, Movimiento «derecho y literatura», Interpretación.

\section{Résumé}

La littérature française paraît avoir abandonné le territoire de la fiction au profit de l'enquête et du témoignage. Les récits judiciaires constituent une importante partie de celle-ci et le rôle de ces récits et des images filmiques dans notre perception du droit ne devrait pas être sous-estimé. Il existe plusieurs manières de concevoir le rapport entre droit et littérature (ou cinéma). La littérature et le cinéma incarnent des personnages d'un procès. Dans une certaine mesure, ils imposent souvent leur interprétation des faits. Enfin, certains procès deviennent des symboles par la littérature et le cinéma ; ainsi, l'écrivain a le pouvoir de modeler notre conception de la justice.

Mots clé : Justice, Droit, Littérature narrative, Mouvement « droit et littérature », Interprétation.

\begin{abstract}
French literature seems to have abandoned the field of fiction to turn to investigation and testimony. Legal narratives are an important part of this, and the role of these narratives and movies in our perception of law might not be underestimated. There
\end{abstract}

\footnotetext{
*Artículo recibido el 25/02/2020, aceptado el 25/10/2020.
} 
are several ways of conceiving the relationship between law and literature (or cinema). Literature and cinema embody the characters of a trial. To a certain extend, they also impose their interpretation of the facts. Finally, some trials have become symbols through literature and cinema; thus, the writer has sometimes the power to shape our conception of justice.

Keywords: Justice, Law, Narrative litterature, «Law and literature» movement, Interpretation.

La littérature française vit actuellement un tournant de son histoire qui la reconduit vers les territoires de l'enquête, du témoignage, de la description de realia savantes et/ou sociales. De cette mutation atteste l'ouvrage de Laurent Demanze Un nouvel âge de l'enquête (2019) ainsi que celui de Marie-Jeanne Zenetti (2015), un peu antérieur. Si la fiction est encore bien présente dans le paysage littéraire, elle se signale souvent elle aussi par un travail assez comparable à celui du romancier du $\mathrm{XIX}^{\mathrm{e}}$ siècle ; recueil de documents en amont, précision des milieux professionnels décrits ${ }^{1}$. De nombreuses soutenances de thèse ${ }^{2}$ indiquent en outre de façon convergente un changement de paradigme des études littéraires favorable au réarrimage de celles-ci aux réalités sociales, scientifiques et épistémologiques. Dans ce panorama des savoirs, le droit ne fait pas exception à la règle au sens où il fait actuellement en France l'objet d'une attention renouvelée dans les relations qu'il entretient avec la littérature. La toute récente parution du collectif Droit et littérature (Baron \& Sarfati, 2019), l'existence à Lille III d'une revue pluridisciplinaire créée et dirigée par Nicolas Dissaux depuis 2017 marquent ainsi l'émergence d'un lien dans lequel la littérature ne se contente pas de figurer des scénarios dans lesquels le droit joue un rôle fondamental (narrations de justiciers, procès célèbres, jurisprudences surprenantes) mais où elle accompagne, et parfois produit des changements de paradigmes comportementaux qui se reflètent dans les décisions de justice.

Pour clarifier le propos, il ne s'agit pas dans cette contribution d'affirmer que les décisions de justice «découleraient» de la lecture de tel texte ou de l'adaptation cinématographique de telle œuvre (ce qui est toujours très difficile à évaluer) mais que le contexte de ces œuvres produit dans la société civile des modifications insensibles de perception qui rendent recevable ou au contraire

\footnotetext{
${ }^{1}$ Voir notamment La clé USB de Jean-Philippe Toussaint (Minuit, 2019), Cora dans la spirale de Vincent Message (Seuil, 2019) ou Le Monde à portée de main, de Maylis de Kerangal (Gallimard, Folio 2018) sur les milieux de la peinture professionnelle.

2 La thèse de Justine Huppe soutenue à Louvain (en 2020) dont le titre est La Littérature embarquée. Réflexivité et nouvelles configurations critiques dans le moment des années 2000 est un exemple de ce changement. L'expression «embarquée » pour désigner la littérature marque le pas avec la doxa sartrienne de la littérature engagée : il s'agit moins de proposer des orientations critiques proches du roman « à thèse » que de prendre acte de mobilisations citoyennes dans lesquelles l'écrivain inscrit son travail, en synergie avec ces mouvements d'opinion.
} 
moins acceptable la conclusion d'un procès ; ces effets de réception des œuvres deviennent des effets de réception de la justice, par rétroaction en quelque sorte (lorsqu'il s'agit de faits passés) mais aussi par projection, lorsqu'une affaire similaire se présente et que la précédente oriente la décision du juge mais aussi l'opinion publique, opinion qui dépend tout autant du travail de la justice que de la nébuleuse de discours tenus autour de ce travail, parmi lesquels ceux des artistes. À la fois rétrospective et projective l'opinio juris se forge non seulement à travers les affaires plus ou moins médiatisées dont le public a connaissance mais également à travers un imaginaire modelé par des récits de fiction documentés ou des documentaires romancés. Robert Cover dans Nomos and Narrative (1983) insiste sur cette perception du droit affinée par la fréquentation des textes littéraires, du cinéma, des séries.

Pour ne prendre que cet exemple, la désobéissance civile de maires (celui de Langouët puis du Perray en Yvelines et d'autres) qui ont interdit par arrêté municipal dans leur commune les pesticides à 150 mètres des habitations, et ce, contre la loi française qui les autorise, est accompagnée d'une sensibilité collective environnementale affinée et cultivée tant par des mouvements d'opinion relayés par les réseaux sociaux que par des récits de fiction postapocalyptiques ${ }^{3}$ qui profilent de manière effrayante le mur écologique dans lequel foncent les sociétés industrialisées du $\mathrm{XXI}^{\mathrm{e}}$ siècle. Si l'écofiction joue un rôle marginal par le nombre de ses lecteurs, en revanche, des séries et reportages qui mettent en scène des désastres écologiques modifient notre appréhension de l'importance de ces mesures et créent des mobilisations citoyennes.

L'exemple canonique de ce type de fait est, dans le mouvement droit et littérature, celui de la parution de César Birotteau de Balzac en 1839, et de sa lecture par des juristes qui entraîna dans le Code civil un assouplissement de la législation relative aux faillites, très sévère envers les débiteurs. Cet exemple illustre de manière directe un pouvoir de la littérature, une puissance de modélisation qui a conduit le droit à une adaptation. Disons pour faire bonne mesure que cette adaptation est relativement rare. Pourtant, ce cas cité par Richard Posner dans son essai traduit en français, Droit et littérature (Posner, 1988) n'est pas unique, et si les arts (littérature, cinéma, séries) n'infléchissent pas le droit de manière frontale, des effets de halo autour d'une affaire peuvent en revanche modeler des convictions ou créer des revirements.

Il convient de rappeler que le mouvement Law and Literature a d'abord pris son essor aux États-Unis sous les auspices d'un courant réaliste du droit qui cultive la fréquentation des textes littéraires dans le but d'améliorer la culture humaniste et sociologique des juges. Une partie non négligeable de cette tendance critique consiste à affiner l'écriture des cas ; c'est alors le droit comme littérature qui est en jeu, dans la mesure où certains récits sont devenus des paradigmes

\footnotetext{
${ }^{3} \mathrm{La}$ 《 postapocalypse » fait même l'objet d'une classification comme sous-genre littéraire dans lequel on trouve des auteurs comme Volodine, E. Faye, N. Ammaniti, V. Bergin, M. Theroux, M. Leboulanger, C. Minard, S. Edgar pour n'en citer que quelques-uns.
} 
jurisprudentiels, exemplaires d'une évolution du droit, mais aussi des sources d'inspiration pour les écrivains.

Le développement actuel en France d'études portant sur droit et littérature ${ }^{4}$ et croisant les méthodes, les manières de lire, les paradigmes critiques de l'une et l'autre discipline nous conduit à nous interroger sur un aspect comparé de l'énonciation judiciaire et de l'énonciation littéraire. Que le droit soit performatif est une évidence. Citons, pour ne prendre que cet exemple, l'article 2 de la Constitution «La langue de la République est le français » : cet énoncé est à la fois constatif, factuel (le français est la langue partagée par la plupart des locuteurs sur le territoire), injonctif (la langue parlée dans les occasions officielles doit être le français à l'exclusion de toute autre) et performatif (affirmer que la langue française est la langue nationale fait exister celle-ci comme langue officielle). Mais si ce pouvoir est inhérent au droit et à l'institution judiciaire, le récit littéraire ou le cinéma sont réputés illustrer, imaginer, reconfigurer à leur manière des réalités, mais en rien les changer. Lorsqu'un scénario romance une affaire, le pouvoir de la fiction réside plutôt dans sa capacité d'instauration ex nihil

- ce que Souriau \& Souriau (1999: 741) désignent ainsi dans son Vocabulaire d'esthétique -mais ne semble pas pour autant agir le réel. Et cependant, certains textes, en particulier lorsqu'ils portent sur l'institution judiciaire, un procès, une enquête qu'ils re-racontent à leur manière, véhiculent des représentations qui peuvent modifier nos opinions. Ainsi le film de Michel Drach Le Pull over rouge paraît en 1979 quasi-simultanément avec l'ouvrage-enquête de Gilles Perrault (1980). Il traite de l'affaire Ranucci, objet de controverses et constitue non seulement un plaidoyer contre la peine de mort mais une œuvre de nature à faire bouger l'opinion publique sur une affaire déjà jugée, d'où la censure dont il a été l'objet, censure municipale, coupures de certaines scènes estimées offensantes et contestées auprès du juge par les parents de la jeune victime.

Dans le dialogue que la littérature entretient avec les savoirs, le droit occupe en effet une place particulière ; tout d'abord parce que l'expertise qu'il suppose, en dépit de l'usage d'un vocabulaire spécialisé des professionnels du droit, est un savoir partageable, partageable au nom de l'adage selon lequel «nul nest censé ignorer la loi ». Mais ce savoir est partageable aussi par ce sentiment intime du juste et de l'injuste, et cet empowerment spécifique qui nous fait mobiliser les connaissances propres à assurer notre défense, le cas échéant, qui rend le citoyen capable d'argumenter face à une décision. Dans L'Amour et la justice comme compétences, texte de rupture avec Bourdieu, Boltanski (1990), en rappelant la connaissance juridique que peuvent mobiliser des acteurs sociaux qui défendent leurs intérêts, nous le rappelle : nous vivons dans des sociétés critiques

\footnotetext{
${ }^{4}$ La parution de la revue de Lille III Droit \& Littérature créée et dirigée par Nicolas Dissaux est un exemple de l'essor de ce tout récent mouvement qui a pris ses lettres de noblesse dans l'Europe francophone belge à travers les travaux de François Ost, Michel de Kerchove (Bruxelles), et en France par les travaux d'Antoine Garapon, Denis Salas et Sandra Travers de Faultrier notamment.
} 
où le citoyen s'empare de la parole ; ainsi, le sociologue n'a pas seul l'apanage du discours réflexif.

C'est ce discours réflexif sur le juste, l'injuste, l'intime conviction qu'interrogera cette contribution à partir de trois modèles et exemples qui prendront appui sur trois caractéristiques du récit judiciaire :

- La posture du témoin auteur ou personnage fictionnel qui, en filtrant le récit, nous confronte à une réactualisation parfois troublante de faits connus ; troublante, mais aussi troublée dans la mesure où dans bien des cas, l'écrivain devient un témoin angoissé du procès et que des processus d'identification se construisent, l'éloignant de la neutralité propre au processus judiciaire.

- La réécriture critique - ou passionnelle - d'un procès pénal par un écrivain ou un cinéaste lui confère parfois un sens nouveau c'est ainsi que, dans un deuxième temps, nous examinerons ce que fait apparaître l'écriture rétrospective: points aveugles de la procédure, fulgurances empathiques, hypothèses, voire réécriture fantasmatique d'autres conclusions possibles.

- Enfin, la prise de position polémique porte une thèse non seulement sur une affaire mais sur le fonctionnement même de l'institution judiciaire et engage la responsabilité de l'écrivain. Un procès peut ainsi devenir emblématique d'une époque, typique d'une situation et, en tant que référence, mobiliser l'énergie interprétative au profit d'une relecture radicale d'un cas, devenir un symbole.

\section{L'artiste comme témoin : un geste d'individualisation}

Dans la réécriture romanesque à laquelle se livre Emmanuel Carrère dans L'Adversaire (2000), l'auteur s'immerge dans l'affaire, côtoie ses acteurs, les questionne à la manière d'un journaliste et rencontre le prévenu, puis le condamné au procès duquel il assiste. Cette manière de documenter un procès se rapproche du New Journalism et de la méthode de cet autre roman célèbre de procès, $D e$ Sang froid de Truman Capote (1965) à quelques exceptions près. Capote délègue la parole dans le roman à un enquêteur, Dewey, qui se fait ouvrir les portes de la maison des Clutter et du pénitencier ; il évite ainsi la première personne. En outre, Truman Capote n'a pas travaillé seul mais l'apport d'Harper Lee qui l'a accompagné dans cette investigation est essentiel ; bien qu'il ait été occulté dans le roman, il suppose une vision de l'affaire qui sapparente à un véritable travail dinstruction à deux voix. Dans ces deux cas, le narrateur s'est imprégné d'une affaire au point que, d'observateur, il devient partie prenante du procès. Par la familiarité qu'il entretient avec les accusés, notamment Perry Anderson - pour lequel il éprouve une compassion tendre -, il réinstruit le procès à sa manière. Quoique non convoqué à la barre, l'écrivain se fait alors témoin de première main, réexamine les pièces du dossier et entend, pour les commenter, les arguments des uns et des autres, à la manière d'un juge ; cette tribune informelle peut poser des problèmes au fonctionnement même de l'institution judiciaire et à la sérénité du débat, mais en sous-estimer l'influence serait une erreur.

Le réalisateur Antoine Raimbault va encore plus loin avec l'affaire Viguier ; lors du premier procès il se rend au tribunal de Toulouse et prend contact 
avec la famille. En effet, le profil très spécifique de l'accusé retient son attention : professeur d'université, grand amateur de romans policiers, et même auteur d'articles sur cette question qui le passionne (Viguier, $2012: 35-46$ ), il déclare la disparition de son épouse mais est immédiatement soupçonné de l'avoir assassinée $^{5}$. Son procès se tient du 20 au 30 avril 2009, puis en appel du 1er au 20 mars 2010 devant les assises du Tarn. Entre temps, le cinéaste a rencontré la famille et convaincu Maître Eric Dupont-Moretti de reprendre l'affaire afin d'optimiser les chances du prévenu de gagner le procès en appel. Son rôle est joué par Olivier Gourmet. Quant au personnage de Nora, incarné par Marina Foïs dans le film que Raimbaut (2019) a beaucoup hésité à réaliser, estimant que sa proximité avec la famille était trop grande, il est entièrement imaginaire et permet à l'auteur de projeter son « intime conviction » sur un tiers.

L'autorisation de la sortie en salles a été l'objet d'un bref feuilleton judiciaire, l'ex-amant de la femme disparue, Olivier Durandet, ayant demandé le retrait du film, au motif que les écoutes téléphoniques reproduites seraient «attentatoires à la vie privée » et par ailleurs, ne présentant que 10 minutes sur 250 heures de captation, seraient toutes à décharge de l'accusé et peu favorables au plaignant ${ }^{6}$.

On ne peut douter que, même si le film ne développe aucune «thèse », Nora - pourtant taclée dans une séquence par Maître Dupont Moretti pour son acharnement - ne soit le porte-parole d'un réalisateur obsédé dans ce dossier par le manque de preuves et surtout la crainte que le tribunal ne voie en Viguier un coupable idéal. L'intime conviction, prévue par l'article 353 du Code pénal est l'unique argument auquel s'accroche Nora, à la fois puissante et fragile car cet article s'adresse, rappelons-le, d'abord au juge, ainsi, dans le roman éponyme de Tanguy Viel (2017) et aux jurés.

Le tournage d'un film, tout comme pour L'Emploi du temps de Laurent Cantet (2001) ou L'Adversaire de Nicole Garcia (2002) n'est donc pas plus anodin que le fait qu'une personnalité du monde littéraire, avec son autorité, prenne la parole au moment d'un procès ${ }^{7}$. Ce phénomène que Laurence Lacour (1993)

\footnotetext{
${ }^{5}$ Le profil intellectuel brillant de l'accusé ne plaide pas en sa faveur, car il rappelle, précisément, des scénarios criminels où un assassin malin joue au chat et à la souris avec l'enquêteur. Le Poète de Michael Connelly est le prototype de ce genre de thriller au cours duquel le policier est susceptible de devenir la prochaine victime, tout comme dans Seven (David Fincher, New Line cinema, 1995), ou encore dans Hangman (film de Johnny Martin, Patriot Pictures, 2017).

${ }^{6}$ La requête formulée auprès de la XVII ${ }^{\mathrm{e}}$ chambre correctionnelle le 19 février 2019 s'appuyait en partie sur un précédent: l'interdiction d'une série d'Arte intitulée Intime conviction sur l'affaire Jean-Louis Muller, et le fait que les téléspectateurs étaient invités à voter (comme cela se pratique aux USA) pour ou contre l'acquittement de l'accusé.

${ }^{7}$ On se souvient de l'article très controversé et factuellement faux de Marguerite Duras au moment de l'affaire Grégory. Le titre racoleur («Sublime, forcément sublime Christine V. » paru dans Libération le 27 juillet 1985) ainsi que la réécriture féministe d'une vie de femme malheureuse en ménage vont provoquer le scandale. Duras a rencontré le juge, convaincu de la culpabilité de la mère et renforcé dans son jugement par l'exaltation de l'écrivain. Laurence Lacour, alors jeune journaliste, fait le point sur cet emballement médiatico-judiciaire.
} 
décrivait au moment de l'affaire Grégory comme la fascination trouble de l'écrivain et du juge - à l'occasion d'un article très controversé de Duras - est toujours présent, et ce, d'autant plus si le propos de l'écrivain et du cinéaste s'inscrit dans une grande proximité chronologique avec l'affaire instruite. Les émotions croisées des artistes, des acteurs judiciaires et des familles atteignent alors leur apogée.

Mais la confrontation de ces cas nous enseigne un autre type de proximité ; dans son essai intitulé Le Fait divers et ses fictions, Frédérique ToudoireSurlapierre (2019) observe une réévaluation de la littérature judiciaire, comme si le mauvais goût associé au fait divers et sa spectacularisation médiatique étaient mis de côté au profit d'un autre regard que porte sur lui la littérature et le cinéma. Le fait qu'un artiste s'empare d'un fait divers efface-t-il magiquement la composante voyeuriste d'un tel geste ? On peut en douter parfois. Cependant, l'écrivain ne se substitue pas à la justice mais fait valoir un autre point de vue ; les déterminismes sociaux en jeu dans une affaire, par exemple, sa composante affective, ou l'histoire dans laquelle se trouve prise le jugement (multiplication d'affaires du même type, précédents judiciaires, disposition de l'opinion, famille des accusés).

En outre, le défi de la littérature consiste à singulariser le fait criminel dont les journaux offrent une vue schématique : «Ce serait peut-être là ce que nous attendons de la littérature, qu'elle transforme l'extrême singularité du fait, mais sans jamais l'effacer, en l'accentuant plutôt, en quelque sorte, visant l'universel ou du moins capable de rejoindre l'autre dans sa propre singularité » (Pontalis, 2011 : 95). Promesse d'une signification, la littérature (comme le cinéma) arracherait ainsi le fait divers à sa contingence, lui restituant son contexte et restituant à la victime son individualité, écrasée par la description scientifique (notamment la description médico-légale) de sa mort lorsqu'il s'agit d'un homicide.

Ainsi, à propos de l'affaire Laetitia, Ivan Jablonka (2016) qui consacre un documentaire-fiction à ce crime très médiatique donne un condensé de ce que peut l'écrivain, sociologue en l'occurrence, face à la parole des experts qui a paradoxalement tendance à abstraire le crime : «Les médecins légistes se sont succédé à la barre, et il a fallu s'approprier les termes qui mettent à distance et déréalisent, "chronologie des lésions traumatiques", "mécanisme de serrage"," appareil ostéo-cartilagineux du cou " »(Jablonka, $2016: 322)$. Cela ne rend compte en rien de l'angoisse qu'a pu être celle de l'adolescente prise au piège d'un tueur, ni de ce que fut sa vie d'avant, ni des raisons qui l'ont poussée à faire confiance à un homme dangereux. La tâche de l'écrivain serait alors triple, selon l'auteur.

D'abord comprendre l'affaire, saisir ses enjeux individuels et collectifs dans les domaines policier, judiciaire et médiatique. Indiquer en quoi le fait divers est un symptôme [...] d'une sensibilité, d'un rapport aux normes.

Ensuite, ouvrir l'affaire, montrer qu'elle n'est pas réductible à un crime, qu'elle renvoie à quelque chose de plus large. Éclairer un état de la société, distinguer un moment, saisir 
des représentations, des discours, des conflits...

Enfin, dissiper l'affaire. Oublier la fin pour libérer la victime de sa mort, pour la restituer à elle-même. [...] Au lieu de dédaigner le fait divers comme le symbole du mauvais goût populaire ou la marotte d'un journalisme dégradé, rappelons-en la potentialité démocratique : il émeut les gens, mais surtout, il nous parle d'eux (Jablonka, $2016: 349$ ).

Si une telle déclaration d'intention ne statue pas sur le mystère du geste criminel lui-même et de ce que Ricœur dans Soi-même comme un autre (1990) nomme l'ascription, soit notre capacité à attribuer un acte à une personne de façon vraisemblable, en replaçant son geste dans une chaîne causale, il restitue du moins à la victime son humanité. Cette empathie que Martha Nussbaum ${ }^{8}$ (2015) place au cœur de la vocation du mouvement «droit et littérature», soit la capacité de la littérature à nous mettre à la place d'autrui, est particulièrement présente dans la plupart des textes où le cinéaste ou l'écrivain assistent au procès, en rencontrent les acteurs et se forgent une opinion sur eux.

Le fait que Truman Capote ait été dans la quasi-incapacité d'écrire après le manuscrit de De Sang froid - l'affaire engloutissant littéralement sa vocation littéraire - est un symptôme. L'identification de l'expérience paternelle d'Emmanuel Carrère à celle qu'a pu vivre Jean-Claude Romand est un autre exemple, très poignant, de cette proximité dérangeante, comme le souligne $\mathrm{F}$. Toudoire -Surlapierre (2019), ainsi l'incipit de L'Adversaire résonne t-il de manière particulièrement étrange: «Le matin du 9 janvier 1993, pendant que JeanClaude Romand tuait sa femme et ses enfants, j'assistais avec les miens à une réunion pédagogique à l'école de Gabriel, notre fils aîné. Il avait cinq ans, l'âge d'Antoine Romand. Nous sommes ensuite allés déjeuner chez mes parents et Romand chez les siens, qu'il a tués après le repas »(Carrère, $2000: 7$ ). La froideur du constat et le parallèle créent chez le lecteur un malaise, l'horreur de la comédie jouée aux proches et particulièrement aux enfants, certes, mais aussi quelque chose de plus complexe qui est la possibilité d'une empathie. L'évocation un peu plus loin de la «tendresse pelucheuse»des enfants et des batailles d'oreillers renvoie à une expérience commune, ébranle le lecteur parce qu'elle suppose une intimité possible, celle de chacun d'entre nous, intimité scandaleuse avec l'auteur d'un crime monstrueux. De cette proximité, Emmanuel Carrère ne fera pas mystère dans L'Adversaire (2000), multipliant les parallèles avec sa vie, et s'interrogeant sur ses propres affects. Il imagine la façon dont les choses auraient pu tourner autrement, par exemple au moment où le prévenu rate son examen de médecine, si Romand avait avoué à Luc - son meilleur ami - son imposture, au lieu de s'inventer un lymphome. L'identification avec Romand est

${ }^{8}$ Il va de soi que bien des œuvres ne s'inscrivent pas dans ce modèle idéal que profile la proposition théorique de Nussbaum ; il existe des œuvres qui font l'apologie du crime, d'autres qui exacerbent la haine. Dans La Connaissance de l'écrivain, publié en 2006, Bouveresse nuance cette approche quelque peu irénique des rapports entre droit et littérature. 
quasi-totale lorsque l'auteur se glisse dans ses pensées et fantasme ses motivations:

Avouer un lymphome à la place d'une imposture revenait pour lui à transposer en termes compréhensibles par les autres une réalité trop singulière et personnelle. Il aurait préféré souffrir pour de bon du cancer que du mensonge car le mensonge était une maladie, avec son étiologie, ses métastases, ses risques de métastases, son pronostic vital réservé -, mais le destin a voulu qu'il attrape le mensonge et ce n'était pas sa faute s'il l'avait attrapé (Carrère, 2000 : 82).

Outre la «vision avec» que suppose ce monologue de l'écrivain (de Romand en débat obscur avec ses motivations), plusieurs éléments attirent l'attention; décrire le mensonge comme une maladie n'est-il pas par avance exonérer son auteur des conséquences de ce mensonge? La supposition comportet-elle une part d'ironie, une allusion à l'auto-aveuglement de Romand? Comment interpréter le «ce n'était pas sa faute », tout autant qu'après l'annonce du verdict et de la durée de la peine le «Si tout se passe bien" ${ }^{9} »$ qui accompagne le souhait d'une durée minimale de prison, à la toute fin du récit (Carrère, 2000 : 204).

Emmanuel Carrère donne un élément de réponse à la fin d'un chapitre relatant le procès et l'attitude de la mère de Florence, prostrée et en proie à une terrible souffrance. Voulant lui manifester sa solidarité, Carrère recule et rappelle au lecteur la place qu'il occupe dans le dispositif judiciaire, qui ne lui donne pas le droit de faire un geste en sa direction:

Ce n'est pas à elle et aux siens que j'avais écrit, mais à celui qui avait détruit leurs vies. C'est à lui que je croyais devoir des égards parce que, voulant raconter cette histoire, je la considérais comme son histoire. C'est avec son avocat que je déjeunais. J'étais de l'autre côté (Carrère, 2000 : 47).

Raconter suppose ainsi choisir un point de vue ; ce lieu commun de la théorie littéraire prend un sens très particulier dans ce contexte de récit judiciaire où la focale détermine la perception des actes et oriente l'opinion des lecteurs. Mais c'est aussi dans ce cas précis d'un don qu'il s'agit; en doublant la parole judiciaire (qui assigne un lieu à Jean-Claude Romand dans le panthéon des monstres) par la parole littéraire qui lui reconnaît sa complexité d'être humain, Carrère engage un travail imaginaire qui consiste à donner un visage au coupable.

\section{Réécritures judiciaires, mises en abyme et retournements}

Dans les exemples précédents, l'écrivain, contemporain d'un fait divers, double la parole du juge, sa propre intervention dans une affaire pouvant être de nature à en modifier la perception ; sa responsabilité pénale est, d'une certaine manière engagée (en particulier lorsque des informations confidentielles de l'instruction ne doivent pas être divulguées). Raimbaut s'est intéressé au procès

${ }^{9}$ « Si tout se passe bien, il sortira en 2015, âgé de 61 ans ». 
Viguier et a pris contact avec ses acteurs au moment de l'appel, par exemple.

Mais l'écrivain ou le cinéaste peuvent se réemparer d'un procès passé et imposer une lecture à telle enseigne que c'est l'œuvre de fiction qui va devenir la référence collective. Ce retournement est présent dans le cadre même du film Une intime conviction; Magalie Flores-Lonjou, qui lui consacre un article, note que la référence à Hitchcock est constante au cours du film de Raimbaut mais que Dupont-Moretti lui-même a dans sa plaidoirie invoqué la référence à deux films : Le Faux coupable (Hitchcock, 1957), pour dire que le doute doit bénéficier à l'accusé, et Fenêtre sur cour (Hitchcock, 1954) pour les cadrages. Elle note en particulier que Nora, la protagoniste, arrivant en retard à une audience, se voit dans l'obligation de suivre celle-ci du couloir, sur une télévision, cette situation renforçant ainsi le dispositif fictionnel d'une mise en abyme du regard sur le prétoire ; significativement ce moment est aussi le plus embarrassant du dossier où Viguier est confronté à la destruction du matelas d'un lit de secours taché, peu après la disparition de sa femme. La présence de la télévision confère alors à cet argument de l'accusation un statut étrange, comme si ce qui apparaissait à l'écran relevait d'une zone indistincte entre fiction et réalité, ce qui sert bien évidemment le propos du film.

Or le personnage de Nora est purement fictionnel, cependant son action auprès de Dupont-Moretti est ce qui va permettre de transformer une enquête à charge, les deux commissaires initialement chargés du dossier étant convaincus de la culpabilité de Viguier, en un procès d'acquittement faute de preuves. Pascale Robert-Diard (2010), journaliste au Monde qui a tenu un blog pendant toute la durée du procès, affirme ainsi, à l'appui des arguments de la défense, que Jacques Viguier n'a pas été poursuivi à cause de ce qu'il est censé avoir fait, mais de ce que beaucoup auraient voulu qu'il soit, à cause de sa singulière personnalité. Ce scénario du procès à charge que Magalie Flores rapproche à juste titre de celui de Garde à vue ${ }^{10}$ prouve son efficacité, dautant que le débat sur la notion même d'intime conviction n'est pas évité par le film.

L'intime conviction est en effet censée découler d'un raisonnement du juré et du juge, fondé certes sur les documents du procès mais aussi sur une certitude plus impalpable qui résulte des impressions produites par les proches, l'accusé luimême, sa personnalité, ou les autres hypothèses qu'on peut formuler sur un crime. Ainsi, Nora est-elle convaincue dès le départ que c'est l'amant, Durandet, qui a assassiné Suzy et tenté de faire accuser le mari. Mais chez Nora, cette certitude est première et emporte tout sur son passage ; elle est le prérequis de sa démarche,

${ }^{10}$ Dans ce film de 1981, un notaire accusé du meurtre de deux fillettes (comme dans l'affaire de Bruay en Artois) est retenu par un policier pugnace qui veut à toute force lui faire avouer les deux crimes. Notable respecté, d'un abord un peu froid, accablé par un témoignage à charge de son épouse sur leur vie intime qui le transforme en coupable idéal, il est finalement relaxé lorsqu'on découvre le cadavre d'une jeune victime, ce qui le disculpe factuellement. L'effet-mémoire de scénarios antérieurs d'acharnement judiciaire n'est sans doute pas pour rien dans l'atmosphère qui a permis dans le procès Viguier le retournement d'une opinion d'abord favorable à la condamnation du prévenu. 
non le résultat de son investigation. Sa dispute avec l'avocat (auquel elle a caché qu'elle avait été jurée dans le premier procès) permet ainsi de mettre au jour le refus de celui-ci de conforter une version partisane, qui ne serait que le double inversé de la condamnation de Viguier par l'opinion publique.

Le cinéma, comme la littérature autorisent ainsi ces effets réflexifs, la monstration d'un débat qui innerve la décision de justice, mais n'est pas toujours accessible, à moins de se procurer les minutes d'un procès. En complexifiant, contre la schématisation journalistique, le cas examiné, le texte, le film produisent des effets de réception qui pluralisent les points de vue, prennent du recul et restituent à une affaire ses prolongements, loin de toute forme de lynchage médiatique, en rappelant constamment le rôle du doute.

Mais l'autorité propre à la littérature et au cinéma ne se réduit pas au constat de cette pluralisation des voix en contexte judiciaire; elle l'historicise et elle prend acte du passage du temps à la manière dont Hayden White problématise le rapport entre science, art et réalité historique :

La science et l'art ont dépassé l'ancienne conception du monde selon laquelle ils devaient fournir la copie littérale d'une réalité supposément statique. Tous deux ont découvert le caractère essentiellement provisoire des constructions métaphoriques auxquelles ils recourent pour rendre compte d'un univers toujours en mouvement. Aussi ont-ils implicitement adopté la conclusion à laquelle Camus était lui-même arrivé: «La question, auparavant, était de savoir si la vie devait avoir un sens pour être vécue. Il est clair maintenant qu'elle sera d'autant mieux vécue qu'elle n'a pas de sens. » On pourrait modifier cette déclaration de la manière suivante: elle sera d'autant mieux vécue qu'elle n'a pas un mais plusieurs sens (White, $2017: 60$ ).

Que la littérature reprenne à nouveaux frais des affaires passées est l'objet même du collectif Incultes en procès paru en 2016 et qui réécrit l'histoire du XX $\mathrm{XX}^{\mathrm{e}}$ siècle à la lumière des grandes et petites affaires qui l'ont jalonnée. Rien n'est exclu dans ce collectif, ni la performance-procès des dadaïstes à Maurice Barrès, ni les procès politiques truqués en contexte totalitaire, ni les affaires criminelles et l'histoire de leur médiatisation. Ce que la littérature permet de faire émerger n'est pas le degré de vérité des faits (qui intéresse la justice) mais la manière dont la perception sociale rejaillit sur l'instruction, dont cette perception aujourd'hui différente nous conduit à voir non le courant de l'histoire mais ce qui va à contrecourant de celle-ci: "Parcours individuels qui sont comme les gouttes d'eau qui participent au fracas de la vague sans être aucunement discernables [...]. Les procès permettent de déconstruire la vague et d'entendre à nouveau le bruit de chaque goutte - ce qui pourrait passer pour l'intention ou l'ambition de beaucoup d'œuvres littéraires » (Incultes, 2016: 8). Arno Bertina, dans la préface de ce collectif, rappelle que Robert Badinter demanda l'abrogation de la peine de mort au moment où la France y était défavorable et, paradoxe, en observant Patrick 
Henry dans la foule déchaînée criant «à mort ! » alors que l'on venait d'arrêter un suspect qui n'était pas l'assassin, rendant ainsi possible une terrible erreur judiciaire.

La littérature et le cinéma n'assument pas nécessairement une position de contre-courant mais en réécrivant les procès ils repotentialisent la décision et en font apercevoir les points aveugles, ou disent purement et simplement le malaise que laisse la manière dont a été conduite une procédure.

Dans ce collectif, Alban Lefranc s'attaque ainsi aux «procès» des membres de la bande à Baader (procès faits en leur absence, prolongés par des propos obscènes visant à discréditer leur parole et à laisser entendre que leurs grèves de la faim furent simulées). Or, ces procès qui ferment, chronologiquement, ce qu'il est convenu d'appeler les «années de plomb» coïncident avec le coming out de Helmut Schmidt qui remercie les juristes allemands de n'avoir pas instruit selon le droit constitutionnel dans l'affaire contemporaine du raid de Mogadiscio. Cette troublante chronologie résonne comme l'aveu de ce qu'est réellement le régime politique de la RFA dans les années 70 ; rien moins qu'une démocratie (Incultes, 2016 : 82). Avant même la capture des membres de la FAR, le sort est joué, rappelle Alban Lefranc, l'affiche les désignant à la vindicte publique rappelle L'Affiche rouge d'Aragon; mines patibulaires, dix-neuf minuscules vignettes surmontées de lettres rouges: «Criminels anarchistes, bande Baader/ Meinhof » (Incultes, $2016: 83$ ) avec une forte récompense à la clef. De sa prison, Meinhof décrira la torture que constitue l'éclairage permanent, l'absence de tout contact humain et son effondrement mental en ces termes:

Sentir ta tête exploser, sentir ta boîte crânienne sur le point d'éclater en morceaux. Sentir ta mœlle épinière te remonter au cerveau à force d'être comprimée. Sentir ton cerveau comme un fruit sec. Se sentir sans cesse et inconsciemment et comme électriquement téléguidée. Sentir qu'on te vole tes associations d'idées. Sentir ton âme pisser de ton corps, comme si tu n'arrivais plus à fixer l'eau. Sentir la cellule bouger (Meinhof apud Incultes, $2016: 86$ ).

Loin de toute interprétation politique c'est le malaise purement physique, celui d'une Allemagne sidérée par une violence d'État sans précédent que met en scène L'Allemagne en automne de Fassbinder; automne du raid aérien et de la découverte quasi-simultanée de trois corps sans vie dans les geôles allemandes; Baader, Ensslin et Raspe. Fassbinder, controversé, et même conspué après la sortie de ce film y extériorise la paranoïa d'un pays entier à travers l'hystérie, le malaise corporel qui s'empare de tous et du cinéaste lui-même. Nulle « leçon » à tirer mais un sentiment continu de suffocation, celui d'un pays écartelé entre l'ultra-violence de groupes d'extrême gauche et la violence non moins radicale d'un État qui agit au mépris du droit. Ce que le cinéma pousse à son paroxysme, c'est à ce moment précis de l'histoire de l'Allemagne la violence occultée par les discours sur le «retour à la démocratie » de l'après-guerre, retour de surface si 
l'on se souvient des derniers mots, nostalgiques, de la mère qui espère l'arrivée providentielle au pouvoir d'un dirigeant autoritaire mais « gentil »... Nazisme qui n'en finit pas de finir et dont les enfants déchirés reproduisent sans fin la violence dont ils sont issus.

Enfin, un procès peut être revu et relu à la lumière de l'histoire ultérieure comme le symptôme d'un changement de paradigme social. Tel est le cas du récit que Julie Bonnie consacre au procès de Bobigny, central dans le processus d'élaboration du texte qui consacre le droit à l'IVG en France. "C'est le procès d'une loi, d'une société » écrit Julie Bonnie, celle d'une France bigote où les «filles-mères » sont parquées dans des établissements où on les traite de manière indigne, sauf si elles sont assez riches pour avorter. Retranscrivant les échanges au tribunal elle y met en relief l'incroyable condescendance du président pour la parole des avocates et témoins, toutes femmes... Première et insupportable asymétrie soulignée par la défense qui poursuit en ces termes : «Le président : Nous ne faisons pas le procès d'une loi. Mme Halimi : Moi, personnellement, je le ferai ». Et plus loin: «Mme Servan Schreiber: C'est l'affaire de toutes les femmes. Le président : Si vous faites venir toutes les femmes ici, on y sera encore avant la fin de l'année » (Incultes, 2016: 77). Julie Bonnie, par-delà l'emballement médiatique et l'intervention de personnalités de monde littéraire et journalistique en faveur de Marie-Claire, fait entendre pour nous la surdité aux faits eux-mêmes ; le viol, le statut de victime de la jeune fille au banc des accusés.

La métaphore auditive est ainsi filée ; celle du grincement («ça grince ! ») et, pourrait-on dire, ce grincement né dans le sillage de 1968 court du procès de Bobigny à \#metoo\# car le point aveugle d'une instruction menée à charge contre la mère de la jeune fille (celle-ci étant mineure) est bien celui de l'agression première :

\footnotetext{
Entendez-vous comme moi que le viol de Marie-Claire n'est pas ou très peu évoqué, que ce soit durant le procès ou dans les médias? Entendez-vous la voix de cette enfant de 15 ans, violée et qui répond au président du tribunal «Je ne veux pas en parler », et le président de passer à autre chose. [...] Daniel P. le violeur n'a JAMAIS été inquiété, ni par l'opinion publique, ni par la justice, alors même que le viol de MarieClaire n'a jamais été remis en question (Incultes, 2016 : 79).
}

Faire entendre ce qui fut inaudible, ou par un changement de point de vue donner voix à ce qui n'a pas de voix est sans doute la vocation de ces textes, qui, déconstruisant, comme le dit Arno Bertina dans l'introduction (Incultes, 2016) la «vague », l'histoire des vainqueurs, revient vers ce qui la constitue ; la multitude des voix discordantes, celles qui racontent une autre histoire ; en l'occurrence l'histoire des enquêtes de moralité que subissaient les femmes portant plainte dans les années 70, les vexations sexistes qualifiées de «gauloiseries» sans conséquence, l'incrédulité de la police, l'arrogance des violeurs et la suspicion des avocats et des juges. 
D'une certaine manière l'institution judiciaire, en condamnant mais en ne faisant pas appliquer les peines aux quatre femmes qui comparaissent au tribunal de Bobigny, en 1972, laisse passer, l'oreille basse, trois années jusqu'au vote de la loi Veil... La loi interdisant l'IVG tranchera le débat, mais le tribunal de Bobigny s'est conformé au droit en vigueur sans mettre en cause un modèle social qui, pourtant, craque de toutes parts depuis les événements de 1968 et atteste un changement de paradigme social profond.

\section{L'institution judiciaire en ligne de mire}

La littérature et le cinéma, certes, singularisent les cas mis en scène, car il y a une forte composante théâtrale au moment du procès mais dans un mouvement symétrique, le filtre par lequel l'institution judiciaire est approchée constitue une métaréflexion sur la manière dont la justice est rendue, ses conditions, ses réussites et ses échecs. La parole de l'artiste pèse de tout son poids lorsque, dénonçant une iniquité, la rendant publique, il fait de son film, de son essai ou de son roman une tribune. Le prototype d'un tel dispositif est sans doute le sort cinématographique de l'Affaire Dreyfus. Lorsque Georges Méliès s'empare du sujet dans l'actualité brûlante de 1899, le procès de Rennes est en cours et Méliès raconte en 11 tableaux l'histoire de Dreyfus, de sa condamnation et de sa dégradation à son arrivée au bagne (Méliès, 1899). La tentative de meurtre sur la personne de Labori, son avocat victime d'un attentat, montre dans quel climat passionnel se déroule alors l'affaire et ce film muet (qui est sans doute le premier film politique en France) lui vaudra des lettres d'insulte et des menaces des antidreyfusards.

En s'attachant à un tel sujet, Roman Polanski (2019) ne risque pas grand chose tant le consensus est fait autour d'une vérité qui était gênante dans le contexte antisémite qui fut celui des premières années du $\mathrm{XX}^{\mathrm{e}}$ siècle, mais qui, s'étant imposée par les faits eux-mêmes, un siècle plus tard, devient le prototype même de l'erreur judiciaire délibérée. Que le réalisateur ait voulu en outre se victimiser en faisant de ce film le porte-drapeau de sa cause d'artiste accusé d'abus sexuels et s'étant constamment soustrait à la justice est un autre débat.

Pour revenir au film de Méliès, absolument remarquable pour l'époque sur le plan technique, il suit d'à peine un an le premier procès et est contemporain du procès de Rennes. Le pathos n'est pas ménagé, comme le rappelle Sylvie Humbert dans l'article qu'elle consacre à la caméra dans le prétoire (Humbert, 2018 : 51-70). La dureté de la vie au bagne, les retrouvailles avec Lucie au bout de cinq ans, les conditions indignes dans lesquelles Dreyfus est traduit en justice une seconde fois, livré à la curée des journalistes, debout alors qu'il est amaigri par sa déportation face au général Mercier assis (Humbert, 2018 : 54-55). Mais la scène la plus choquante est sans doute celle où Labori, joué par Méliès lui-même, tombe dans la rue, agressé et blessé par un homme qui prend la fuite, alors que les piétons passent leur chemin. On ne secourt pas un dreyfusard. Ces notations ajoutées à la puissance (et la rareté) des images cinématographiques à cette période vont avoir un grand retentissement populaire ; joué dans des foires et des 
salles de cinéma, il déclenche les foudres de la censure qui s'abat sur le film par des arrêtés préfectoraux. Son influence sera néanmoins considérable car elle aura montré ce qu'est une instruction à charge, lorsque dès la première scène, un pistolet est tendu à l'accusé pour qu'il se donne la mort évitant ainsi un procès.

Dans ce qu'elle a de plus haïssable c'est ainsi l'injustice délibérée, calculée, une justice à la botte des militaires et du pouvoir politique qui ne veulent pas qu'un Juif accède à des postes de responsabilité dans l'armée qui est dénoncée, un an après le pamphlet de Zola, en plein cœur de cette mini-guerre civile. Le fait qu'aujourd'hui ce texte et ce film soient invoqués constamment lorsque la partialité du tiers judiciaire est mise en cause est symptomatique du fait que ces œuvres sont devenues des filtres interprétatifs incontournables. Il semblerait que dans l'imaginaire commun ils aient eu un retentissement bien plus important que la grâce du président Loubet puis la réhabilitation tardive du capitaine Dreyfus.

Autant pourrait-on en dire des œuvres qui ont ponctué la marche de la communauté noire vers l'égalité de droits dans les États du Sud de l'Amérique, et leur reconnaissance en Europe ; de la «négritude » exaltée dans les poèmes de Sédar Senghor à des œuvres-totem, c'est par la littérature et le cinéma que des discours émancipateurs ont trouvé leur public. La reprise par des artistes (sous forme de biopics par exemple) de la vie des figures héroïques du mouvement (Rosa Parks, Martin Luther King) n'échappe pas à la règle faisant de leur combat politique une épopée. L'acquisition de droits n'est ainsi pas le seul enjeu de ces combats mais ils portent aussi sur la dignité des personnes et cet argument est sans doute bien plus puissant car il concerne chacun de nous.

L'identification par la fiction joue alors le rôle qu'attribue Martha Nussbaum à la littérature dans Les émotions démocratiques (2011). Par l'empathie nous traversons les identités imaginaires que les récits de fiction mettent à notre disposition, constituant ainsi une sorte de stock d'émotions. Mais le rôle de cellesci ne se limite pas à un partage instantané et éphémère de sensibilité ; car à travers elles, c'est une forme de citoyenneté qui émerge.

Particulièrement lorsqu'il s'agit de contextes judiciaires, les enjeux qu'exposent les récits, qu'ils soient documentaires ou imaginaires, constituent un catalyseur d'affects d'autant plus puissants que la capacité d'identification est grande. Mais ces émotions ne sont pas pour autant irrationnelles; comme l'observe Daniel Kahneman dans Système 1/système 2 ; les deux vitesses de la pensée (2012), les émotions primaires constituent la base de ce qui oriente nos décisions et notre action à un niveau plus profond, celui d'émotions secondaires durables qui est celui des valeurs que nous construisons et auxquelles nous sommes attachés. Ainsi, conformément à ce que note Aristote dans L'Éthique à Nicomaque, la colère est-elle une authentique passion citoyenne et non une folie passagère : elle nous permet ainsi de manifester ce que nous attendons (et ce que nous refusons) dans la vie publique. Or, l'injustice subie, mais aussi celle dont nous sommes spectateurs est sans doute l'expérience la plus propre à susciter des 
passions, passions du droit selon l'expression de François Ost (2018), désir de droit.

Une œuvre littéraire en faveur de l'égalité entre communautés aux ÉtatsUnis va particulièrement marquer sa génération, moins parce qu'elle montre une injustice que parce que l'un de ses héros, l'avocat Atticus Finch qui défend un prévenu noir fait preuve en toute circonstance d'une équité sans faille. Il s'agit de Ne tirez pas sur l'oiseau moqueur de Harper Lee, publié en 1960 : ce roman remporte immédiatement un immense succès public et obtient le prestigieux prix Pulitzer.

Rappelons quelques éléments de l'intrigue: le récit est porté par Scout, la fille d'un avocat veuf qui vit avec son frère et son père dans une petite ville du Sud des États-Unis. La communauté se passionne pour une histoire qui oppose une famille blanche pauvre dont la fille porte plainte pour viol, à un jeune noir, l'accusé, qui travaille comme ouvrier agricole au profit de ces poor white trash campagnards qui le haïssent. C'est en réalité la jeune fille qui a fait des avances à Robinson, sans succès, et qui se venge de cette manière ${ }^{11}$. Son père, Bob Ewell qui la soutient - s'en prendra aux enfants de l'avocat de la partie adverse, Scout et Jem, qui seront sauvés de justesse par leur jeune voisin. Les haines raciales exacerbées par cette affaire conduisent les deux enfants dont le père défend le jeune garçon noir à suivre de près cette affaire et à la raconter de leur point de vue.

Bien avant le verdict, les insultes pleuvent sur Atticus Finch, mais un épisode secondaire pose aux yeux des lecteurs l'assise du personnage, et justifie sa réputation d'équité. Une vieille femme acariâtre, Madame Dubose, traite Finch d' «ami des nègres » en invectivant ses enfants de son perron, alors qu'ils se rendent à une kermesse. Au retour, Jem, le jeune frère de Scout, après avoir ruminé sa vengeance se jette sur les parterres de fleurs de la vieille dame et les massacre. Son père, averti de la situation, au lieu d'attiser la haine contre une ennemie politique contraint l'enfant à tenir compagnie à la vieille dame malade et à lui faire la lecture pendant une durée fixée à un mois. Cette punition ${ }^{12}$ restaure un lien abîmé et, choisissant d'oublier l'offense qui lui a été faite, Finch manifeste un respect égal à ceux qui le soutiennent et à ses détracteurs. Il se montre moins dans cet épisode avocat que juge équitable.

Le juge et les jurés mettront d'ailleurs plus de temps à trancher l'affaire qu'il plaide que d'ordinaire ; ils délibèreront vraiment, alors que, la parole d'un jeune noir ne valant rien dans les États du Sud des années 50-60, le procès aurait dans d'autres circonstances expédié en prison le jeune condamné sans examen. Ce que met en scène ce texte est précisément cet idéal d'equity que promeut la Théorie de la justice de John Rawls (1971) ; examen sans préjugé du cas soumis,

${ }^{11}$ Ce scénario rappelle dans un tout autre contexte celui des Risques du métier d'André Cayatte, tourné en 1967, qui met en scène un instituteur accusé de viol par une jeune fille qu'il a punie, puis d'autres du village, par imitation.

${ }^{12}$ Qui ressemble fort à ce que nous nommerions aujourd'hui une mesure de justice restaurative. 
souci de réparation par la justice des inégalités de toute nature, refus de s'en remettre à la solution paresseuse du précédent judiciaire.

Il en va tout simplement de la crédibilité de l'institution judiciaire dans ces récits véritables ou fictifs mais inspirés de multiples faits réels; les sources connues du roman de Harper Lee (1960) ne laissent aucun doute sur le fait qu'elle a eu connaissance de cas similaires, rassemblant plusieurs affaires en une seule. En outre sa passion pour les affaires criminelles est, nous l'avons vu, partagée avec Truman Capote, autre grande figure littéraire qui fut aussi son ami d'enfance.

Mais ce que peut mettre en discussion une œuvre c'est non seulement le fonctionnement de la justice, mais un point de droit. Emmanuel Carrère ne s'est pas seulement intéressé au procès pénal avec L'Adversaire, mais à des aspects plus modestes de la pratique du droit; l'endettement des ménages devient un phénomène endémique en France dans les années 90, en plein essor du néolibéralisme. Les contrats opaques de sociétés de crédit, la facilité avec laquelle on peut emprunter et le peu de garanties demandées précipitent de nombreux ménages dans la spirale du surendettement dont ils ne sortent bien souvent que par un passage devant le juge. D'autres vies que la mienne (Carrère, 2009) s'attache au quotidien des juges confrontés à des dossiers de ménages en cessation de paiement, et d'emblée, les héros de l'histoire, Etienne et Juliette, agissent à contre-courant de ce qui leur a été enseigné à l'école, se démarquant ainsi de pratiques routinières pour porter attention au substrat humain des affaires qui leur sont confiées.

Etienne se souvient ainsi qu'à $1^{\prime} E N M^{13}$ on lui avait présenté très brièvement les surendettés comme des consommateurs abrutis dont la condamnation était la seule réponse sensée à leur imprévoyance. Sa pratique l'entraîne à déchiffrer les stratégies de sociétés de crédit, la violence de leurs méthodes de recouvrement et l'opacité de leurs contrats ; avec les armes du droit, il combat ces pratiques et rend avec équité la justice en ne privant pas de remboursement les petites sociétés qui s'effondreraient avec des impayés: il ne s'agit pas d'une pratique empathique au sens psychologique du terme, d'une approche «sensible », mais d'un discernement bienvenu qui n'abîme pas le lien social et ne conforte pas les pratiques de vente agressives et opaques. Cette évolution des pratiques judiciaires qui tient compte de la vulnérabilité des justiciables est ici rendue visible au public par la littérature mais aussi le cinéma ; presque contemporain de la parution du roman, le film de Philippe Lioret Toutes vos envies sort en 2011.

La discussion de points de droit dans le film comme dans le roman joue parallèlement un rôle d'information sur la législation en vigueur, de la loi Scrivener ${ }^{14}$ qui n'a jamais été réellement appliquée à la loi Hamon et à l'existence

\footnotetext{
${ }^{13}$ L'École Nationale de la Magistrature, créée en 1958, se situe à Bordeaux.

${ }^{14}$ Cette loi $\mathrm{n}^{\circ} 78622$ du 10 janvier 1978 s'applique aux crédits à la consommation et aux crédits pour travaux d'un montant de 200 euros à 75000 euros. Elle a été amendée par une disposition de 2011 suite à la réforme du crédit à la consommation pour une durée supérieure à trois mois. Cette
} 
d'un fichier centralisé des crédits.

En l'occurrence, dans le maquis des règles juridiques, ces récits de vies constituent parfois des boussoles, car, loin de se contenter de représenter les conditions dans lesquelles s'applique le droit, ils sont des carrefours de voix diverses et des occasions de penser nos propres pratiques en les replaçant dans un contexte légal. En dépit du fait qu'il évoque des personnes ayant réellement existé (auxquelles l'auteur demande parfois l'autorisation de rapporter tel épisode), le roman de Carrère possède des traits fictionnels. Fiction documentaire, ou document fictionnalisé se croisent alors dans une pratique qui a pour but moins de représenter que de problématiser notre rapport au droit.

Prenant acte de cette tendance profonde de la littérature française contemporaine (et peut-être d'une grande partie des littératures européennes d'aujourd'hui), le cinéma documentaire s'est récemment enrichi d'un film sur l'institution judiciaire. Rendre la justice ${ }^{15}$, sorti en novembre 2019 sur les écrans, rend visible le travail des juges et des magistrats (procureurs notamment), tant au niveau européen qu'au niveau national. Cette grande machine, perçue comme effrayante, impersonnelle, broyeuse, est exposée à travers ses acteurs, de l'école où ils sont formés à Bordeaux aux plus hautes fonctions qu'ils peuvent exercer dans la magistrature. Ce sont des vies qui sont racontées et non la puissance d'une institution qui est exposée (même si la caméra s'arrêtant sur l'architecture souligne la majesté des lieux de justice).

Les histoires se croisent, de cette magistrate nommée en Guyane qui découvre dès les premières affaires à traiter qu'elle est perçue comme une blanche venue juger des populations métissées à ce jeune procureur d'origine nordafricaine insulté par ceux qui, se prévalant d'une origine ethnique commune, le perçoivent comme un traître à sa communauté, jusqu'à cette juge qui, ayant demandé un certificat médical pour une garde partagée avec un père soupçonné d'alcoolisme, apprend que celui-ci s'est donné la mort la veille de la confrontation. Mais il y a aussi ce procureur confronté aux corps déchiquetés de victimes d'un attentat, et cette autre juge qui évoque la difficulté à faire parvenir jusqu'au prétoire des affaires financières douteuses, faute de moyens suffisants pour démasquer des montages complexes. À travers leurs témoignages se dessine alors une histoire bien différente du droit français, de la manière dont il est appliqué.

loi impose aux sociétés de crédit de formaliser par un contrat une offre préalable soumise à un délai de rétractation, d'une part, d'autre part, de préciser sur l'offre les conditions du crédit, le TEG actualisé, la durée, et le coût total avec et sans assurance.

La loi Hamon 2014-344 du 7 mars autorise les actions de groupe lorsqu'il y a préjudice au consommateur.

${ }^{15}$ Rendre la Justice de Robert Salis, sorti le 13 novembre 2019, date anniversaire des attentats du Bataclan et des cafés environnants, sort au moment où les luttes sociales se multipliant en France, l'incompréhension et le conflit entre justice, police et citoyens est à son comble depuis la crise des gilets jaunes, qui a débuté en novembre 2018. On peut émettre l'hypothèse que ce film répond à une intention politique de réparation d'une image écornée de l'institution. 
Il ne s'agit pas là de storytelling, même si l'émotion est présente (et contenue) à chaque moment de témoignages parfois étonnants mais d'une incarnation du droit, incarnation qui constitue l'apport essentiel du cinéma et de la littérature à l'histoire d'une institution. Car seule, sans doute, cette incarnation est-elle de nature à modifier l'appréhension commune, l'image que peuvent se forger les acteurs de la société civile de ce qui régit leurs propres vies.

\section{RÉFÉRENCES BIBLIOGRAPHIQUES}

BARON, Christine \& Judith SARFATI (2019) : Droit et littérature. Nîmes, SFLGC, Éd. Lucie.

BolTANSKI, Luc (1990) : L'Amour et la justice comme compétences. Paris, Métailié.

CAPOTE, Truman (1972 [1965]) : De Sang froid. Paris, Gallimard (Folio).

CARRÈRE, Emmanuel (2000) : L'Adversaire. Paris, POL.

CARRÈRE, Emmanuel (2009) : D'Autres vies que la mienne. Paris, POL.

COVER, Robert (1983): «Nomos and Narrative». Harvard Law Review, 97 [The Supreme court, 1982].

Demanze, Laurent (2019) : Un Nouvel âge de l'enquête. Paris, Corti (Les Essais).

DIARD, Pascale (2010) : «Procès Viguier, affaire publique». Le Monde (Chroniques judiciaires). 16 mars. URL : https://www.lemonde.fr/societe/2010/03/16/dans-leproces-viguier-l-amant-raconte-le-mari-volage-et-epouse-delaissee_1319827.3224.html.

FLORES-LONJOU, Magalie (2019): "D'Une femme disparaît à Fenêtre sur cour en passant par la fabrique du Faux coupable dans Une Intime conviction d'Antoine Raimbault ». Politeia, 36, 342-359.

HARPER LEE, Nelle (2006 [1960]) : Ne tirez pas sur l'oiseau moqueur. Traduction de Isabelle Stoïanov. Paris, Grasset (Le Livre de poche).

HuMbert, Sylvie (2019) : «La Caméra dans le prétoire, miroir de la société ». Les Cahiers de la justice, 1:1 (Séduction et peur des images), 51-70. URL: https://doi.org/10.3917/cdlj.1901.005.

INCUltes (2016) : Incultes en procès. Coordination de Arno Bertina. Paris, Édition Incultes (Dernière marge).

JABLONKA, Ivan (2016) : Laetitia. Paris, Seuil (Librairie du XXI ${ }^{\mathrm{e}}$ siècle).

KAHNEMAN, Daniel (2012) : Système 1/système 2 ; les deux vitesses de la pensée. Paris, Flammarion (Essais).

LACOUR, Laurence (1993): Le Bûcher des innocents: l'Affaire Villemin, coulisses, portraits, preuves, engrenages, correspondances, choses vues. Paris, Plon.

NuSSBAUM, Martha (2011) : Les émotions démocratiques. Traduction de Solange Chavel. Paris, Climats.

Nussbaum, Martha (2015) : L'Art d'être juste. Traduction de Solange Chavel. Paris, Climats. 
OST, François (2018): Droit et passions du droit. Bruxelles, Académie Royale de Belgique .

PerRault, Gilles (1980) : Le Pull-over rouge. Paris, Livre de poche.

PONTALIS, Jean-Bernard (2011) : Un jour, le crime. Paris, Gallimard (Folio).

POSNER, Richard (1996 [1988]) : Droit et littérature. Paris, Odile Jacob.

RAWLS, John (1971) : A Theory of Justice. Cambridge, Harvard University Press.

RICEUR, Paul (1990) : Soi-même comme un autre. Paris, Seuil (L'ordre philosophique).

SOURIAU, Etienne \& Anne SOURIAU (1999): Vocabulaire d'esthétique. Paris, Presses Universitaires de France (Quadrige).

TOUDOIRE-SURLAPIERRE, Frédérique (2019) : Le Fait divers et ses fictions. Paris, Minuit, (Paradoxe).

VIEL, Tanguy (2017) : Article 535 du Code pénal. Paris, POL.

VIGUIER, Jacques (2012) : «Vote et légitimité dans L'Homme qui tua Liberty Valance ». Politeia, 21 (Le vote à l'écran), 35-46.

WhITE, Hayden (2017): L'Histoire s'écrit. Traduction et présentation de Philippe Carrard. Paris, Éditions de la Sorbonne (Libre cours).

ZENETTI, Marie-Jeanne (2015) : Factographies. L'enregistrement littéraire à l'époque contemporaine. Paris, Garnier.

\section{RÉFÉRENCES FILMOGRAPHIQUES}

CAYATTE, André (1967) : Les Risques du métier. Gaumont International.

DRACH, Michel (1979) : Le Pull-over rouge. Gaumont et Port-Royal Films.

GARCIA, Nicole (2002) : L'Adversaire. Sarde Films.

HITCHCOCK, Alfred (1954) : Fenêtre sur cour. Paramount Pictures.

HiтCHCOCK, Alfred (1957) : Le Faux coupable. Warner Bros.

LIORET, Philippe (2011): Toutes vos envies. Production de Philippe Lioret et Marielle Duigout.

MÉLIÈS, Georges (1899) : L'Affaire Dreyfus. Star Film.

MiLler, Claude (1981) : Garde à vue. Les Films Ariane - TF1 Films Production.

POLANSKI, Roman (2019) : J'Accuse. Légende films - Gaumont Film Company.

RAIMBAUT, Antoine (2018) : Une Intime conviction. Delante Productions.

SALIS, Robert (2019) : Rendre la justice. Eden Films - Ladybirds Films - France 2 Cinéma. 